\title{
Ordering in Solving Systems of Equations ${ }^{1)}$
}

\author{
Kun Jiang, Xiao-Shan Gao \\ Institute of System Sciences, AMSS, Academia Sinica \\ Beijing 100080, P.R. China \\ E-mail: (kjiang, xgao)@mmrc.iss.ac.cn
}

\begin{abstract}
To use a "good" variable order is one of the effective ways to prevent the occurrence of large polynomials in an elimination method. In this paper, we present an algorithm of $O\left(n^{3}\right)$ to find such a "good" order using algorithms from graph theory. Based on this algorithm, we present a new version of Wu-Ritt's zero decomposition algorithm, which allows different orders for different triangular sets in the decomposition formula.
\end{abstract}

\section{Introduction}

It is well known that Wu's method is very fast in proving geometry theorems[?]. Many difficult theorems whose traditional proofs need an enormous a mount of human intelligence, such as Feuerbach's theorem, Morley's trisector theorem, etc., can be proved by computer programs based on Wu's method within seconds[?].

This method first introduces a coordinate system so that the geometric entities and relations are turned into a system of equations, and then decides in an algorithmic manner whether the conclusion is valid on the solutions under some non-degenerate conditions. Therefore, Wu's method is essentially based on an algebraic elimination theory. One of the reason behind the success of Wu's method is that we may choose a nice order for the points in a geometry theorem. This order will lead to a variable order which will make the elimination procedure easy.

It is well-known that finding a "good" variable order is one of the effective ways to prevent the occurrence of large polynomials in a general elimination algorithm. So it is an effective strategy to find a nice order of variables before starting an elimination process. For instance, when using Wu's method to prove geometry theorems, one difficulty is the problem of reducibility. It has been noticed that deliberate choice of variable order can help avoiding or at least lessening reducibility difficulties [?].

There are two main approaches to find variable orders: one is at geometric level, the other is at algebraic level. Finding variable order in geometric level is actually a geometric method of equation solving, in which a complicated diagram is translated into a constructive form (using ruler and compass) which is easy to compute. This process is also called geometric constraint solving[?, ?, ?, ?]. A degree of freedom analysis method is proposed to find a nice order for geometric objects [?]. Finding the variable order in algebraic level was not studied extensively. In [?], a heuristic method is proposed to find variable order mainly according to

Thhis work is supported by a National Key Basic Research Project (NO. G1998030600) and by the CNSF under an outstanding youth grant (NO. 69725002) 
the degree of variables. This is a local method in that it does not provide global structure of the equation system.

In this paper, a graph approach is developed by analyzing the structural solvability of a system of equations. With this approach we can obtain independent solvable parts in the structural sense and a solving order among these parts. Consequently, the solving order of variables in the system of equations can be obtained. This algorithm is especially effective for large equation systems with hundreds of variables, like the systems from CAD [?].

As an application, we give a new version of Wu-Ritt's decomposition algorithm. Since Wu-Ritt's zero decomposition repeatedly does elimination for polynomial sets, we may use the graph algorithm to present a new version of Wu-Ritt's zero decomposition algorithm, which allows different order for different triangular sets in the decomposition formula.

\section{DM-Composition of Bipartite Graph}

We first recall the definition of the bipartite graph and related knowledge that play a central role in our approach. For an in-depth study, see [?, ?]. Let us assume that the system of equations to be solved is as follows:

$$
\left\{\begin{array}{c}
f_{1}\left(x_{1}, x_{2}, \ldots, x_{n}\right)=0 \\
f_{2}\left(x_{1}, x_{2}, \ldots, x_{n}\right)=0 \\
\vdots \\
f_{n}\left(x_{1}, x_{2}, \ldots, x_{n}\right)=0
\end{array}\right.
$$

where each $f_{i}$ is a polynomial. In the above system of equations, it is possible that some variables disappear in some equations.

Let $G=(V, E)$ be a directed graph with vertex set $V$ and edge set $E$. For two vertices $\mathrm{u}$ and $\mathrm{v}$, we denote as " $u \longrightarrow v$ on $G$ " (or simply, $u \longrightarrow v$ ), if and only if there exists a directed path from $\mathrm{u}$ to $\mathrm{v}$ on $G$.

Two vertices $\mathrm{u}$ and $\mathrm{v}$ belong to the same strongly connected component if and only if $u \longrightarrow v$ and $v \longrightarrow u$. The vertex set $V$ is partitioned into strong connected components $V_{i}$, each of which determines the vertex induced subgraph $G_{i}=\left(V_{i}, E_{i}\right)$ of $G$, also called a strong component of $G$. Partial order $\prec$ can be defined on the family of strong components $G_{i}=\left(V_{i}, E_{i}\right)$ of $G$, or, in other words, on the family of subsets $V_{i}$ of $V$, by

$$
V_{i} \prec V_{j} \Longleftrightarrow v_{j} \longrightarrow v_{i} \text { on } G \text { for some } v_{i} \in V_{i} \text { and } v_{j} \in V_{j} \text {. }
$$

We also write $G_{i} \prec G_{j}$ if $V_{i} \prec V_{j}$.

A bipartite graph is a directed graph denoted by $B=\left(V^{+}, V^{-} ; E\right)$ where the vertex set consists of two disjoint parts $V^{+}$and $V^{-}$, and the edges are directed from $V^{+}$to $V^{-}$. For $W^{+}\left(\subset V^{+}\right)$, we use $\Gamma\left(W^{+}\right)$to denote the set of vertices in $V^{-}$adjacent to the vertices in $W^{+}$. A Matching $M$ on $B$ is a subset of $E$ such that no two edges in $M$ share a common vertex incident to them. A matching of maximum cardinality $|E|$ (the number of edges in $M)$ is called a maximum matching. In case $\left|V^{+}\right|=\left|V^{-}\right|$, a matching that covers all the vertices is a complete matching. For $M, \partial^{+} M$ stands for all initial vertices of all edges in $M$ and $\partial^{-} M$ stands for all terminal vertices of all edges in $M$.

Based on the maximal matching, a unique decomposition of a bipartite graph into partially ordered irreducible bipartite subgraphs can be defined. This decomposition, due to 
Dulmage-Mendelssohn, will be referred to as the DM-composition, and the irreducible components $B_{i}=\left(V^{+}, V^{-} ; E_{i}\right)$ as DM-components. An example [?]is illustrated in Figure 1 and Figure 2. An algorithm for the DM-decomposition is given below, where $G_{M}=\left(V^{+} \cup V^{-}, \tilde{E} ; S^{+}, S^{-}\right)$is the auxiliary graph associated with a matching $M$ and is defined as follows:

$$
(v, w) \in \tilde{E} \Longleftrightarrow(v, w) \in E \text { or }(w, v) \in M,
$$

and $S^{+}=V^{+} \backslash \partial^{+} M$ and $S^{-}=V^{-} \backslash \partial^{-} M$ are called the entrance and exit.

\section{Algorithm 1: The DM-decomposition of $B=\left(V^{+}, V^{-} ; E\right)$}

step 1 Find a maximum matching $M$ on $B=\left(V^{+}, V^{-} ; E\right)[?, ?]$.

step 2 Let $V_{0}=\left\{v \in V^{+} \cup V^{-} \mid w \longrightarrow v\right.$ on $G_{M}$ for some $w$ in $\left.S^{+}\right\}$.

step 3 Let $V_{\infty}=\left\{v \in V^{+} \cup V^{-} \mid v \longrightarrow w\right.$ on $G_{M}$ for some $w$ in $\left.S^{-}\right\}$.

step 4 Let $V_{i}(i=1, \ldots, r)$ be the strongly connected components of the graph obtained from $G_{M}$ by deleting the vertices of $V_{0} \cup V_{\infty}$ and the edges incident thereto.

step 5 Define the partial order $\prec$ on $\left\{V_{i} \mid i=0,1, \ldots, r, \infty\right\}$ as follows:

$$
V_{i} \prec V_{j} \Longleftrightarrow v_{j} \longrightarrow v_{i} \text { on } G_{M} \text { for some } v_{i} \in V_{i} \text { and } v_{j} \in V_{j} \text {. }
$$

In this algorithm, a maximum matching can be found in $O\left(|E|\left(\min \left\{\left|V^{+}\right|,\left|V^{-}\right|\right\}\right)^{1 / 2}\right)$ $[?, ?]$, where $|E|$ is the number of the edges and $\left|V^{+}\right|,\left|V^{-}\right|$the number of $V^{+}, V^{-}$respectively. The execution time of step 2 and step 3 are direct proportional to $|E|$. Finding the strongly connected components can be done in $O\left(\left(\left|V^{+}\right|+\left|V^{-}\right|\right)^{2}\left(\left|V^{+}\right|+\left|V^{-}\right|-1\right)\right)$. Therefore, the time complexity of the Algorithm 1 is $O\left(\left(\left|V^{+}\right|+\left|V^{-}\right|\right)^{3}\right)$.

Note that the decomposition does not depend on the choice of maximum matching $M$. We call the component $V_{0}\left(\right.$ or $\left.B_{0}\right)$ the minimal inconsistent part, the component $V_{\infty}\left(\right.$ or $\left.B_{\infty}\right)$ the maximum inconsistent part, and the rest the consistent part. The DM-decomposition reveals the structure of a bipartite graph with reference to the maximum matching on it.

\section{Generate Triangular Blocks}

The basic idea of solving an equation system is to triangularize it. Namely, the original system is transformed into a triangular form that can be easily solved by sequentially solving a series of univariate polynomial equations. The variable order is crucial for the triangularization. In general, a good order means that the original equations are already in a triangular block form, like the one shown in (2). In order to get a good order, we make use of the bipartite graph theory to analyze the structural solvability of the system of equations and decompose the system into some subsets, which are independent solvable parts named triangular blocks. With the triangular blocks and the partial order we can get a order of variables. This order is especially useful in solving the systems consisting of hundreds of variables and equations. In practice, this kind of equations often occurs in $C A D$ [?]. 


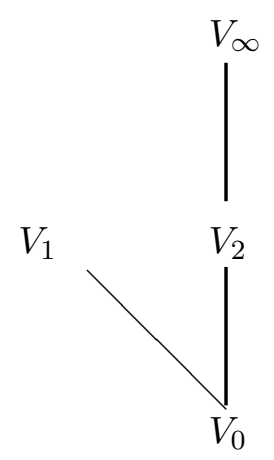

Fig. 1. DM-decomposition of a bipartite

Fig. 2. Partial order graph

$$
\left\{\begin{array}{l}
f_{1}\left(x_{i_{1}}, \ldots, x_{i_{t_{1}}}\right)=0 \\
f_{t_{1}}\left(x_{i_{1}}, \ldots, x_{i_{t_{1}}}\right)=0 \\
f_{t_{1}+1}\left(x_{i_{1}}, \ldots, x_{i_{t_{1}}}, x_{i_{t_{1}}+1}, \ldots, x_{i_{t_{2}}}\right)=0 \\
f_{t_{2}}\left(x_{i_{1}}, \ldots, x_{i_{t_{1}}}, x_{i_{t_{1}}}+1, \ldots, x_{i_{t_{2}}}\right)=0 \\
\quad \vdots \\
f_{t_{b_{-1}}+1}\left(x_{i_{1}}, \ldots, x_{i_{t_{1}}}, \ldots, x_{i_{t_{b-1}}+1}, \ldots, x_{i_{t_{b}}}\right)=0 \\
f_{t_{b}}\left(x_{i_{1}}, \ldots, x_{i_{t_{1}}}, \ldots, x_{i_{t_{b-1}}}+1, \ldots, x_{i_{t_{b}}}\right)=0
\end{array}\right.
$$

\subsection{Generating Triangular Blocks}

We only consider a system of equations in the standard form (1), where $x_{i}$ are variables and $f_{i}$ are equations. Let $F$ be the set of equations, $X$ the set of all variables appearing in the equations and $E$ the set of edges defined by the pairs $\left(f_{i}, x_{i}\right)$ with $f_{i} \in F$ and $x_{i} \in X$ such that the variable $x_{i}$ appears in the equation $f_{i}$. Then $B=(X, F ; E)$ is the bipartite graph associated with the system of equations (see Example 1). This bipartite graph is called the bipartite graph representation of the system of equations.

Example 1 Consider the following system of equations:

$$
f_{1}\left(x_{1}, x_{2}, x_{3}\right)=0, f_{2}\left(x_{2}, x_{3}, x_{5}\right)=0, f_{3}\left(x_{1}, x_{4}\right)=0, f_{4}\left(x_{3}\right)=0, f_{5}\left(x_{4}, x_{5}\right)=0 .
$$

The bipartite graph representation is shown in Figure 3 [?].

\section{Algorithm 2: Ordering in solving system of equations}

Input: A system of equations.

Output: The solving order of variables of the system of equations.

step 1 Generate the bipartite graph representation $B=(X, F ; E)$ for the system of equations. 
Fig. 2. the Bipartite graph representation

step 2 Process the DM-decomposition of $B=(X, F ; E)$ with Algorithm 1.

step 3 If $V_{0} \neq \varnothing$ then the system of equations is under-determined and goto step 5.

step 4 If $V_{\infty} \neq \varnothing$ then the system of equations is over-determined. One or more equations in $V_{\infty}$ should be deleted by the users to obtain a well-determined problem and goto step 5 .

step 5 Output the variables in $\left\{V_{i} \mid i=0,1, \ldots, r, \infty\right\}$ according to the partial order defined on it.

According to the order of the variables, we can quickly solve the system of equations. In this algorithm, step $\mathbf{1}$ can be done in $O(|E|)$ steps. The complexity of step $\mathbf{5}$ is $O(|X|)$. Since $|E|$ is at most $O\left(\left(\left|V^{+}\right|+\left|V^{-}\right|\right)^{2}\right)$, the complexity of Algorithm 2 is $O\left(\left(\left|V^{+}\right|+\left|V^{-}\right|\right)^{3}\right)$,

Remark The system of equations is structurally solvable iff both $V_{0}$ and $V_{\infty}$ are the empty set. A DM-component $V_{i}, 1 \leq i \leq r$, in the consistent part corresponds to a triangular block that is structurally solvable and can not be further decomposed. It can be solved independently if the values of all the variables belonging to $V_{j}$ such that $V_{j} \prec V_{i}$ are determined. The triangular blocks corresponding to the inconsistent parts, $V_{0}$ and $V_{\infty}$, are not solvable. The problem corresponding to $V_{0}$ is under-determined (i.e. has more variables than equations) and that to $V_{\infty}$ is over-determined (i.e. has fewer variables than equations).

Notice that the variable order obtained is a partial order. To give a total order of variables, we may use the method presented in [?] to give variable order from the same block.

Example 2 Consider the following CAD problem [?]: a lathe traveling steady (Figure 4). In Figure 4 , a point is indicated by $p_{i}$ and a line by $l_{i}$. Lines that are constrained to be horizontal or vertical are indicated by $h_{i}$ or $v_{i}$.

The constraints are: on $\left(p_{1}, v_{1}\right)$,on $\left(p_{1}, h_{1}\right)$, on $\left(p_{2}, h_{1}\right)$, on $\left(p_{2}, v_{5}\right)$, on $\left(p_{3}, h_{2}\right)$, on $\left(p_{3}, v_{1}\right)$, on $\left(p_{4}, h_{2}\right)$, on $\left(p_{4}, v_{2}\right)$, on $\left(p_{5}, h_{2}\right)$, on $\left(p_{5}, v_{3}\right)$, on $\left(p_{6}, h_{2}\right)$, on $\left(p_{6}, v_{4}\right)$, on $\left(p_{7}, h_{3}\right)$, on $\left(p_{7}, v_{3}\right)$, on $\left(p_{8}, h_{3}\right)$, on $\left(p_{8}, v_{4}\right)$, on $\left(p_{12}, l_{4}\right)$, on $\left(p_{12}, h_{5}\right)$, on $\left(p_{9}, h_{4}\right)$, on $\left(p_{9}, v_{2}\right)$, on $\left(p_{9}, l_{1}\right)$, on $\left(p_{15}, h_{7}\right)$, on $\left(p_{15}, l_{1}\right)$, on $\left(p_{16}, l_{1}\right)$, on $\left(p_{16}, l_{2}\right)$, on $\left(p_{17}, l_{2}\right)$, on $\left(p_{10}, h_{4}\right)$, on $\left(p_{10}, v_{5}\right)$, on $\left(p_{11}, h_{4}\right)$, on $\left(p_{11}, v_{6}\right)$, on $\left(p_{13}, v_{5}\right)$, on $\left(p_{13}, h_{6}\right)$, on $\left(p_{14}, v_{6}\right)$, on $\left(p_{14}, h_{6}\right)$, on $\left(p_{13}, l_{3}\right)$, on $\left(p_{17}, l_{3}\right)$, distance $\left(v_{1}, v_{5}\right)$, distance $\left(v_{2}, v_{5}\right)$, distance $\left(v_{2}, v_{3}\right)$, distance $\left(v_{4}, v_{5}\right)$, distance $\left(v_{5}, v_{6}\right)$, distance $\left(h_{1}, h_{2}\right)$, distance $\left(h_{2}, h_{3}\right)$, distance $\left(h_{1}, h_{5}\right)$, distance $\left(l_{1}, l_{4}\right)$, distance $\left(p_{12}, h_{4}\right)$, distance $\left(p_{12}, h_{7}\right)$, 
Fig. 3. A sketch figure of the lathe traveling steady

distance $\left(p_{15}, p_{16}\right)$, distance $\left(h_{6}, h_{4}\right)$, distance $\left(p_{16}, p_{17}\right)$, angle $\left(l_{4}, h_{5}\right)$, angle $\left(l_{1}, l_{2}\right)$. A distance between two lines also implies parallelism. In order to make the diagram having a fixed position, we specify the coordinates of the point $p_{1}$ by adding a constraint knowncoordinates $\left(p_{1}\right)$. With a coordinate system, all the constraints can be transformed into a system of equations. We use $x=a_{i}, y=b_{i},\left(x_{i}, y_{i}\right)$, and $y=m_{i} x+n_{i}$ to represent a vertical line $v_{i}$, a horizontal line $h_{i}$, and a point $p_{i}$, and a line $l_{i}$ respectively. The system of equations contains 55 variables and 55 equations. Applying Algorithm 2 to the system, we can obtain an variable order: $x_{1} ; y_{1} ; a_{1} ; m_{4} ; m_{1} ; m_{2} ; a_{5} ; a_{2} ; a_{3} ; a_{4} ; a_{6} ; b_{1} ; b_{2} ; b_{3} ; b_{5} ; y_{2} ; y_{3} ; y_{4} ; y_{5} ; y_{6}$; $y_{7} ; y_{8} ; x_{2} ; x_{3} ; x_{4} ; x_{5} ; x_{6} ; x_{7} ; x_{8} ; y_{12} ; x_{13} ; x_{14} ; x_{10} ; x_{11} ; x_{9} ; b_{4} ; b_{7} ; b_{6} ; y_{9} ; y_{10} ; y_{11} ; y_{13} ; y_{14}$; $y_{15} ; x_{12}, n_{4}, n_{1} ; x_{15}, x_{16}, y_{16} ; n_{2} ; x_{17}, y_{17} ; m_{3}, n_{3}$, where we use ";" to separate the blocks.

\subsection{Wu-Ritt's Zero Decomposition Algorithm with Mixed Order}

Wu-Ritt's zero decomposition algorithm is used to decompose the zero set of a set of polynomial equations into the union of zero sets of polynomial equations in triangular form [?]. Precisely speaking, we have

Theorem(Wu-Ritt's Zero Decomposition Algorithm) For any given polynomial set $P S$, we may find a series of polynomial sets $T S_{i}, i=1, \ldots, d$ in triangular form under one variable order such that

$$
Z \operatorname{ero}(P S)=\bigcup_{i} Z \operatorname{ero}\left(T S_{i} / J_{i}\right)
$$

where $J_{i}$ is the product of initials of the polynomials in $T S_{i}$. This method repeatedly uses a triangular procedure called well-ordering principle which takes a polynomial set $P S$ as input and find a triangular polynomial set $T S$ of $P S$ [?].

Since the triangular procedure is sensitive to variable order, we propose that each time before using this triangular procedure we generate a new variable order with our algorithm. In this way, we have a different version of the decomposition algorithm.

Theorem (Wu-Ritt Zero Decomposition with Mixed Order) For a polynomial set $P S$, we may find a series of variable orders $o_{i}$ such that

$$
Z \operatorname{ero}(P S)=\bigcup_{i} Z \operatorname{ero}\left(T S_{i} / J_{i}\right)
$$


where each $T S_{i}$ is in triangular form under variable order $o_{i}$ and $J_{i}$ is the initial product of $T S_{i}$.

As an example, let us consider the $P 3 P$ problem [?]: "Given the relative spatial locations of three control points, and given the angle to every pair of control points from an additional point called the Center of Perspective $\left(C_{P}\right)$, find the lengths of the line segments joining $C_{P}$ to each of the control points."

Let $P$ be the Center of Perspective, and $A, B, C$ the control points. Let $|P A|=x,|P B|=$ $y,|P C|=z$ and $p=\cos \alpha, q=\cos \beta, r=\cos \gamma$. Introducing the following parameters: $A=\frac{b^{2}+c^{2}-a^{2}}{2}, B=\frac{c^{2}+a^{2}-b^{2}}{2}$, and $C=\frac{a^{2}+b^{2}-c^{2}}{2}$, we may find the following equation set $P S$

$$
\left\{\begin{array}{c}
x^{2}+y z p-z x q-x y r-A=0 \\
y^{2}+z x q-y z p-x y r-B=0 \\
z^{2}+x y r-y z p-z x q-C=0 .
\end{array}\right.
$$

We need only to find solutions under $G \neq 0$, where

$$
G=x y z\left(p^{2}-1\right)\left(q^{2}-1\right)\left(r^{2}-1\right)\left(B x_{1}+C\right)\left(A x_{2}+C\right)\left(A x_{3}+B\right)
$$

where $x_{1}, x_{2}, x_{3}$ are new introduced auxiliary variables. Applying Wu-Ritt's decomposition method [?] to $(P S)$, we obtain a triangular set $T S_{1}$

$$
\left\{\begin{array}{l}
f_{1}=I_{1}^{2} x^{8}+C_{16} x^{6}+C_{14} x^{4}+C_{12} x^{2}+C_{10} \\
f_{2}=x S_{2} y-C_{20} \\
f_{3}=x S_{3} z-C_{30}
\end{array}\right.
$$

where $S_{2}=\left(I_{1} I_{2} I_{3}\right)^{4}, S_{3}=\left(I_{1} I_{2} I_{4}\right)^{4}, I_{1}=p^{2}+q^{2}+r^{2}-2 p q r-1, I_{2}=C q(r p-q)+$ $B r(r-p q), I_{3}=C p(r q-p)+A r(r-p q), I_{4}=B p(r q-p)+A q(q-r p)$. Detailed coefficients of $f_{1}, f_{2}, f_{3}$ are omitted. Let $J=x I_{1} I_{2} I_{3} I_{4}$. From Wu-Ritt's Decomposition Theorem, we have

$$
Z \operatorname{ero}(P S / G)=\operatorname{Zero}\left(T S_{1} / J_{1}\right) \cup \bigcup_{i=1}^{4} Z \operatorname{Zero}\left(\left(P S, I_{i}\right) / I_{1} \ldots I_{i-1} G\right)
$$

where $T S_{1}=\left\{f_{1}, f_{2}, f_{3}\right\}, J_{1}=J G$. The first part of the above decomposition is the main component for the $P 3 P$ equation system and the last four zero sets correspond to the special or degenerate cases.

The three degenerate cases $I_{i}=0, i=2,3,4$ are similar. Let use consider $I_{2}=0$. Using the Gröbner basis program of Maple, we find that the polynomials set $\left(P S, I_{2}\right)$ can be decomposed into two branches:

$$
\begin{array}{r}
Z \operatorname{Zero}\left(\left(P S, I_{2}\right) / I_{1} G\right)=\bigcup_{i=1}^{2} Z \operatorname{Zero}\left(G S_{2 i} / I_{1} G\right) \text { where } \\
G S_{21}=\left\{\begin{array}{l}
I_{2} \\
(p q-r) z^{2}+r C \\
r y-q z \\
(p q-r)\left(x^{2}-2 q x z-A\right)-C q p
\end{array}\right.
\end{array}
$$




$$
G S_{22}=\left\{\begin{array}{l}
I_{2} \\
r I_{1} x^{2}+C p(q-r p)+A r\left(1-p^{2}\right) \\
r I_{1}(p q-r)\left(y^{2}-2 r x y\right)-C_{0} \\
(r p-q) z+(p q-r) y+\left(r^{2}+q^{2}-2 p q r\right) x .
\end{array}\right.
$$

Here $C_{0}=C(p r-q)\left(q^{3}-2 p q^{2} r+q r^{2}-q+r p\right)+A r(p q-r)\left(r^{2}+q^{2}-2 p q r\right)$. Before using Wu-Ritt's algorithm to $G S_{21}$ and $G S_{22}$, we may notice that $G S_{21}$ and $G S_{22}$ are already in triangular form under the variable orders: $z<y<x$ and $x<y<z$ respectively. This fact can be easily detected with our graph algorithm. So further decompositions for $G S_{21}$ and $G S_{22}$ should be carried out according to these two variable orders respectively.

\section{Conclusion and Future Work}

We propose an approach to find variable order for solving a system of equations. We make use of the bipartite graph to decompose the system of equations into triangular blocks. At the same time, we also obtain a partial order among these triangular blocks. This partial order may lead to a variable order. Solving a system of equations with the order of variables obtained by our approach can speed up the solving procedure, especially for large equation systems consisting of hundreds of equations.

Our approach will lose effectiveness when all variables dependent on each other. In other words, these equations form one block. An interesting work is to break these blocks into smaller blocks. This has been done successfully for a large class of problems in geometric level $[?, ?, ?, ?]$. In algebraic level, there is still no essential progress.

\section{References}

[1] B. Bruderlin, Using Geometric Rewriting Rules for Solving Geometric Problems Symbolically, Theo. Comp. Sci., 116, 291-303, 1993.

[2] S. C. Chou, Mechanical Geometry Theorem Proving ,D. Reidel Publishing Company, Dordrecht, Netherlands(1988).

[3] X. S. Gao and S. C. Chou, Solving Geometric Constraint Systems I. A Global Propagation Approach, $C A D$, 30(1), 47-54(1998).

[4] X. S. Gao and H. F. Cheng, On the Solution Classification of the "P3P" Problem, Proc. ASCM'98, eds Z. B. Li, pp. 185-200, LanZhou University Press(1998).

[5] C. Hoffmann, Geometric Constraint Solving in $R^{2}$ and $R^{3}$, in Computing in Euclidean Geometry, World Scientific, pp. 266-298(1995).

[6] R. S. Latheam and A. E. Middleditch, Connectivity Analysis: a Tool for Processing Geometric Constraints, CAD, 28(11), 917-928, 1994.

[7] H. Li and M. Cheng, Automated Ordering for Automated Theorem Proving in Elementary Geometry-Degree of Freedom Analysis Method, MM Research Preprints No. 18, pp.84-98(1999).

[8] L. Lovász and M. D. Plummer, Maching Theory, Annals of Discrete Mathematics 29, NorthHolland, Amsterdam(1986).

[9] K. Murota, Systems Analysis by Graphs and Matroids Algorithms and Combinatorics 3, SpringerVerlag, New York(1987).

[10] J. Owen, Algebraic Solution for Geometry from Dimensional Constraints, in ACM Symp. Solid Modeling, ACM Press, pp. 397-407(1991). 
[11] D. Wang, An Implementation of the Characteristic Set Method in Maple. In: Automated practical reasoning: Algebraic approaches, Springer, New York, pp. 187-201(1995).

[12] W. T. Wu, Basic Principles of Mechanical Theorem Proving in Geometries, Volume I: Part of Elementary Geometries, Science Press, Beijing (in Chinese) 1984. English Translation, Springer, 1994. 